\title{
Residual Strains In Directed Energy Deposition Additive Manufacturing.
}

\author{
Daniel Weisz-Patrault ${ }^{1, a)}$ \\ ${ }^{1 *}$ LMS, CNRS, École Polytechnique, Institut Polytechnique de Paris, F-91128 Palaiseau, France. \\ ${ }^{a}$ Corresponding author: daniel.weisz-patrault@polytechnique.edu \\ URL: www.polytechnique.edu/annuaire/en/users/daniel.weisz-patrault
}

\begin{abstract}
Recently, a fast macroscopic and semi-analytical thermal analysis of Laser Metal Powder Directed Energy Deposition (LMPDED) has been submitted to publication. The effect of various process parameters are taken into account as well as solidification and solid-state phase transitions. One of the main advantage of the proposed approach is that the model is fast enough to simulate the entire process and perform parametric studies or optimization loops. Thus, residual strains induced by temperature variations and phase transitions are easily computed. In addition, transformation induced plasticity is also investigated. Within this framework, temperature control strategies can be optimized.
\end{abstract}

\section{INTRODUCTION}

Laser Metal Powder Directed Energy Deposition (LMPDED) involves injecting a stream of metallic powder that is melted by a laser beam in order to deposit material layer-by-layer on a substrate [1,2]. LMPDED induces very specific temperature history including very high temperature gradients (near the melt pool) and thermal cycling. Both the microstructure formation/evolution (solidification, solid state phase transitions, grain mobility etc.) and the formation of residual stresses are driven by thermal conditions during the process. Therefore, significant efforts have been made to simulate accurately, at the macroscopic scale, both temperature evolutions and solidification kinetics. However, macroscopic modeling of such processes is computationally costly [3-12]. This hinders the development of efficient numerical optimization of process parameters in order to reach microstructure and material properties requirements and avoid residual stress issues (e.g., distortions, cracks, buckling etc.).

Recently, a simplified macroscopic and semi-analytical thermal analysis of LMPDED has been submitted to publication [13]. The approach relies on a semi-analytical solution of the transient non-linear heat conduction problem in a two-dimensional multilayer composite ( $r, z$ radial and vertical coordinates). The derivation of the mathematical solution extends previous analytical thermal analysis on composites (e.g.,[14, 15]). Mathematical developments relies on several Fourier-Bessel series expansions and an alternating algorithm to deal with non-linearity. Numerical tasks mostly consist in computing successive roots (or eigenvalues) of a characteristic function representing boundary conditions. In [13], a 100 layers cylinder has been simulated within around 20 min on a personal computer. Computation time is therefore fairly reduced in comparison with classic numerical schemes based on finite element method or finite difference method.

In this contribution, the model proposed recently in [13] is exploited to investigate residual strains induced by temperature variations and phase transitions. In addition, transformation induced plasticity [16] is also investigated. Various process parameters are tested and residual strains during the process are analyzed.

\section{MODEL STRATEGY AND ASSUMPTIONS}

The model developed in [13] relies on a semi-analytic solution of the unsteady and nonlinear heat equation. The general strategy is summarized in this section. In addition, the proposed solution is obtained by introducing some 
assumptions that are also recalled for sake of clarity. Further details and all mathematical developments are given in [13].

Volumetric heat sources due to the enthalpy changes during phase transitions arise as a right side term in the unsteady heat equation. As these heat sources strongly depends on the temperature field, the heat equation is non linear. This issue is overcome by an alternating scheme. Indeed, the heat equation becomes linear and easier to solve if the right side term is imposed (fixed to a known evolution). Thus, a first estimation of the temperature field is performed with a right side term arbitrarily set to zero. Then, the obtained estimated temperature field is used as inputs of a simple phase transition model based on Johnson-Mehl-Avrami-Kolmogorov (JMAK) equation. Phase proportion rates are then used to compute the right side term of the heat equation and a new estimation of the temperature field is computed on this basis. This procedure is repeated until convergence.

Despite the fact that a nonlinear problem is solved as a succession of linear problems, the unsteady linear heat equation remains difficult to solve analytically mostly because of geometrical complexity and time dependency of the domain. Indeed, as new mass is regularly added to the part during the process, the support domain of the heat equation is strongly time dependent. To overcome these geometrical difficulties, the main assumption is to neglect heat fluxes along the tangent direction of the laser path (denoted by $\chi$ ). That is to say that successive points on $\chi$ can be considered as independent. The validity of this assumption is questionable in the vicinity of the melt pool where temperature gradients are very significant in all directions. However, at the scale of the part, as molten metal is deposited at very high temperature on top of a relatively cold multilayer structure, heat fluxes along the vertical direction prevail.

This assumption leads to consider several independent computations at different positions on the path $\chi$. Each of these computation points consists in multilayer 2D structure in the $(r, z)$ plane, where $r$ is the radial coordinate (thickness direction) and $z$ the vertical coordinate. The number of layers gradually increases as metal deposition goes on. Each computation point is characterized by (i) the radius of curvature of the path $\chi$ at the corresponding position and (ii) the different times of metal deposition on top of the multilayer structure denoted by $\left(t_{1}, \cdots, t_{N+1}\right)$ (where $N$ is the final number of layers). The simulation strategy consists in solving analytically the heat equation for each computation point on each time interval $\left[t_{n}, t_{n+1}\right](1 \leq n \leq N)$. Thus, each computation point requires $N$ sub-computations that are simply connected to each other by setting the initial condition on the time interval [ $\left.t_{n}, t_{n+1}\right]$ as the final condition on the previous time interval $\left[t_{n-1}, t_{n}\right]$. It should be noted that for the same geometry different laser paths and dwell times can be simulated through different $\left(t_{1}, \cdots, t_{N+1}\right)$. The layer thickness $h_{r} \mathrm{~mm}$ and height $h_{z} \mathrm{~mm}$ are fixed parameters.

For sake of simplicity, the power per unit area brought to the system by the radiative term and by the laser is modeled as a power per unit volume. In addition, the gas flow that is used to carry the powder increases significantly the heat transfer coefficient for all layers under the flow. However, the mathematical solution is based on constant heat transfer coefficient (denoted by $H$ ). Thus, temperature losses due to the gas flow are also modeled through the introduction of an additional negative volumetric heat source. On this basis, the unsteady heat conduction equation reads:

$$
\frac{\partial^{2} T^{(i)}}{\partial r^{2}}+\frac{1}{r} \frac{\partial T^{(i)}}{\partial r}+\frac{\partial^{2} T^{(i)}}{\partial z^{2}}-\frac{1}{D} \frac{\partial T^{(i)}}{\partial t}=\frac{Q^{(i)}(t)}{\lambda}
$$

where $T^{(i)}$ is the temperature in the layer $(i), D\left(\mathrm{~m}^{2} \cdot \mathrm{s}^{-1}\right)$ is the thermal diffusivity. In this contribution, material parameters ( $D$ diffusivity and $\lambda$ thermal conductivity) are assumed to be temperature independent and identical in all layers. However, heterogenous and temperature dependent properties are considered in [13]. The volumetric heat source $Q^{(i)}$ contains four contributions: the radiative term, the heat source due to the enthalpy change during phase transition, the volumetric heat source in the top layer of the already existing part due to the laser and the volumetric negative heat source associated to the local increase of the heat transfer coefficient due to the gas flow.

The substrate is modeled in a simplified way. It is assumed that the substrate temperature $T_{\text {sub }}$ is constant on each time interval $\left[t_{n}, t_{n+1}\right]$. The substrate temperature $T_{\text {sub }}$ is updated at the end of the $n$-th sub-computation according to 
the total energy received during the time interval $\left[t_{n}, t_{n+1}\right]$. Thus, boundary conditions read:

$$
\begin{aligned}
& \text { (a): } \begin{cases}\lambda \frac{\partial T^{(i)}}{\partial r}=H\left(T^{(i)}-T_{\mathrm{ext}}\right) & r=R_{\mathrm{inf}} \quad(1 \leq i \leq n) \\
\lambda \frac{\partial T^{(i)}}{\partial r}=-H\left(T^{(i)}-T_{\mathrm{ext}}\right) & r=R_{\mathrm{sup}} \quad(1 \leq i \leq n)\end{cases} \\
& \left(\text { b) : } \left\{\begin{array}{ll}
\lambda \frac{\partial T^{(1)}}{\partial z}=H_{\mathrm{sub}}\left(T^{(1)}-T_{\mathrm{sub}}\right) & z=Z^{(0)} \\
\lambda \frac{\partial T^{(n)}}{\partial z}=-H\left(T^{(n)}-T_{\mathrm{ext}}\right) & z=Z^{(n)}
\end{array}\right.\right. \\
& \text { (c) : }\left\{\begin{array}{lll}
T^{(i)}=T^{(i+1)} & z=Z^{(i)} & (1 \leq i \leq n-1) \\
\frac{\partial T^{(i)}}{\partial z}=\frac{\partial T^{(i+1)}}{\partial z} & z=Z^{(i)} & (1 \leq i \leq n-1)
\end{array}\right.
\end{aligned}
$$

where $Z^{(i)}$ denotes the interfaces in the multilayer structure, $R_{\text {inf }}$ and $R_{\text {sup }}$ denote the inner and outer radius respectively. In addition, $H_{\text {sub }}$ denotes the heat transfer coefficient between the part and the substrate.

The mathematical solution relies on several Fourier-Bessel series expansions. More precisely, a set of basis functions verifying Equation 1 with boundary conditions (Equation 2) are obtained by separation of variables. Then, a function vector space is generated. The solution verifying both boundary conditions and the initial condition is approximated by an orthogonal projection on a linear subspace of finite dimension. The projection involves the introduction of a scalar product for which the basis functions are orthogonal. Numerical tasks are mostly limited to compute successive roots (or eigenvalues) involved in the basis functions, which makes this approach very efficient with respect to the computation cost.

\section{RESIDUAL STRAINS}

Thermal expansion and volume variations during phase transitions are modeled by a hydrostatic strain (proportional to the identity tensor) denoted by $\varepsilon^{\text {thm }}$ (where thm stands for thermo-metallurgical ). Since these contributions only depend on temperature and phase proportions evolutions, the proposed model enables to directly compute the associated eigenstrain as follows:

$$
\dot{\varepsilon}^{t h m}=\sum_{k=2}^{N} \frac{1}{3}\left(\frac{\rho_{1}(T)}{\rho_{k}(T)}-1\right) \dot{X}_{k}+\left(\alpha_{1}+\sum_{k=2}^{N} X_{k}\left(\alpha_{k}-\alpha_{1}\right)\right) \dot{T}
$$

Where $T$ is the temperature, $X_{k}$ the $k$-th phase proportion, $\alpha_{k}$ the thermal expansion coefficient of the $k$-th phase and $\rho_{k}$ the density of the $k$-th phase.

However, deviatoric strains (i.e., traceless) also arises. For instance, transformation induced plasticity (TRIP) classically refers to deviatoric plastic strains observed during phase transitions that occur under mechanical loads (that can be lower than the yield stress). Simple models [16] have been obtained by homogenization procedure and enable to deal with multiphase mixtures. For instance the transformation induced plasticity strain rates denoted by $\dot{\varepsilon}^{t p}$ reads:

$$
\dot{\varepsilon}^{t p}=\sum_{k=2}^{N} \frac{S_{k}}{\sigma_{k}^{Y}} \frac{\sigma_{k}^{Y}-\sigma_{k}^{e q}}{\mu_{k} \xi_{k}} \dot{X}_{k}+ \begin{cases}0 & \text { if } \quad\left|\widetilde{\varepsilon}^{t h m}\right|<\frac{\Delta \sigma^{Y}}{\zeta} \\ -\frac{3\left|\widetilde{\varepsilon}^{t h m}\right| S_{1}}{\sigma_{1}^{Y}} \ln \left(\frac{\Delta \sigma^{Y}}{\left|\widetilde{\varepsilon}^{t h m}\right| \zeta}\right) \sum_{m=2}^{N} \dot{X}_{m} & \text { if } \quad X \leq \frac{\Delta \sigma^{Y}}{\zeta\left|\widetilde{\varepsilon}^{t h m}\right|} \leq 1 \\ -\frac{3\left|\widetilde{\varepsilon}^{t h m}\right| S_{1}}{\sigma_{1}^{Y}} \ln (X) \sum_{m=2}^{N} \dot{X}_{m} & \text { if } \quad X>\frac{\Delta \sigma^{Y}}{\zeta\left|\widetilde{\varepsilon}^{t h m}\right|}\end{cases}
$$

where the parent phase is indexed by 1 and product phases are indexed by $k \geq 2, S_{k}$ is the stress deviator in the $k$-th phase, $\sigma_{k}^{Y}$ is yield stress of the $k$-th phase, $\sigma_{k}^{e q}$ is the von Mises equivalent stress of the $k$-th phase, $X$ is the sum of all the product phases $\left(X=1-X_{1}\right), \lambda_{k}, \mu_{k}$ are the Lamé's coefficients of the $k$-th phase and:

$$
\Delta \sigma^{Y}=\sqrt{\left(\sigma_{1}^{Y}\right)^{2}-\left(\sigma_{1}^{e q}\right)^{2}} \text { and } \zeta=\frac{\left(3 \lambda_{1}+2 \mu_{1}\right) 2 \mu_{1}}{\lambda_{1}+2 \mu_{1}}
$$


and where $\widetilde{\varepsilon}^{\text {thm }}$ represents an approximation of the strain history:

$$
\widetilde{\varepsilon}^{\text {thm }}=\sum_{k=2}^{N} \frac{X_{k}}{1-X_{1}}\left(\frac{1}{3}\left(\frac{\rho_{1}(T)}{\rho_{k}(T)}-1\right)+\frac{\rho_{1}(T)}{\rho_{k}(T)}\left(\alpha_{k}-\alpha_{1}\right)\left(T-T_{0}\right)\right)
$$

The main difficulty is that the transformation induced plastic strain rate depends on the stress field, leading to a nonlinear mechanical problem (for instance [17]), which is still in development. In this contribution, to estimate the impact of transformation induced plasticity without performing mechanical computations, various uniform stress fields are tested. Only the evolution of the eigenstrain (or residual strain) during the process is analyzed. The analysis of residual stresses would necessitate mechanical computations that are not performed in this contribution.

\section{CONCLUSION}

The proposed approach provides a very fast tool to simulate temperature and phase transitions in directed energy deposition additive manufacturing. The entire process can be simulated and parametric studies or optimization loops can be performed. The present work investigates residual strains arising during the process, which can be used as inputs in a mechanical model in order to compute residual stresses induced by temperature variations and phase transitions. Thus, the proposed model, coupling thermal analysis and phase transitions, is part of a more general model, still in development, including mechanical computations and transformation induced plasticity.

\section{REFERENCES}

[1] W. E. Frazier, Journal of Materials Engineering and Performance 23, 1917-1928 (2014).

[2] T. DebRoy, H. Wei, J. Zuback, T. Mukherjee, J. Elmer, J. Milewski, A. M. Beese, A. Wilson-Heid, A. De, and W. Zhang, Progress in Materials Science 92, 112-224 (2018).

[3] L. Wang, S. D. Felicelli, and J. E. Craig, "Thermal modeling and experimental validation in the lens tm process," in 18th Solid Freeform Fabrication Symposium. Austin, TX (2007), pp. 100-111.

[4] H. Liu, "Numerical analysis of thermal stress and deformation in multi-layer laser metal deposition process," Ph.D. thesis, Missouri University of Science and Technology 2014.

[5] G. Marion, G. Cailletaud, C. Colin, and M. Mazière, "A finite element model for the simulation of direct metal deposition,” in International Congress on Applications of Lasers E Electro-Optics, Vol. 1 (LIA, 2014), pp. 834-841.

[6] E. R. Denlinger and P. Michaleris, Additive Manufacturing 12, 51-59 (2016).

[7] J. Smith, W. Xiong, J. Cao, and W. K. Liu, Computational mechanics 57, 359-370 (2016).

[8] T. Keller, G. Lindwall, S. Ghosh, L. Ma, B. M. Lane, F. Zhang, U. R. Kattner, E. A. Lass, J. C. Heigel, Y. Idell, et al., Acta materialia 139, 244-253 (2017).

[9] Q. Chen, G. Guillemot, C.-A. Gandin, and M. Bellet, Additive Manufacturing 16, 124-137 (2017).

[10] D. Zhang, Z. Feng, C. Wang, Z. Liu, D. Dong, Y. Zhou, and R. Wu, Journal of Thermal Spray Technology 26, 831-845 (2017).

[11] C. Baykasoglu, O. Akyildiz, D. Candemir, Q. Yang, and A. C. To, Journal of Manufacturing Science and Engineering 140, p. 051003 (2018).

[12] M. Biegler, B. Graf, and M. Rethmeier, Additive Manufacturing 20, 101-110 (2018).

[13] D. Weisz-Patrault, Submission (2019).

[14] F. De Monte, International Journal of Heat and Mass Transfer 46, 1455-1469 (2003).

[15] D. Weisz-Patrault, International Journal of Heat and Mass Transfer 104, 595-606 (2017).

[16] D. Weisz-Patrault, Journal of the Mechanics and Physics of Solids 106, 152-175 (2017).

[17] D. Weisz-Patrault and T. Koedinger, Applied Mathematical Modelling 60, 18-33 (2018). 